\title{
Inbreeding Depression Analysis for Yield and Some of its Associated Characters in Late Sown Condition in Bread Wheat (Triticum aestivum L.em. Thell)
}

\author{
Ritu Choudhary*, Hoshiyar Singh, Chhagan Lal and Darshita Bhat
}

Seed Testing Laboratory, Chittorgarh, (Raj) India

*Corresponding author

\section{A B S T R A C T}

\section{Keywords}

Inbreeding

,Depression,

Bread wheat,

Yield,

Late sown

Article Info

Accepted:

15 June 2018

Available Online:

10 July 2018
In order to study inbreeding depression for yield and some of its associated characters in bread wheat (Triticum aestivum L.em. Thell) over the environments, the present experiment was carried out performing diallel analysis following Griffing (1956) Method II Model I. Ten genetically diverse parents were selected and crossed in half diallel fashion excluding reciprocals. These parents were evaluated along with their $45 \mathrm{~F}_{1}$ 's and $45 \mathrm{~F}_{2}$ ' $\mathrm{s}$ in a randomized block design with 3 replications over three environments namely, normal sown $\left(E_{1}\right)$, late sown $\left(E_{2}\right)$ and very late sown $\left(E_{3}\right)$ environments created by using three different dates of sowing. In the present experiment significant inbreeding depression was reported for different traits in all the three environments. Even though, none of the cross displayed significant inbreeding depression in desired direction for all the characters and in all the environments but HD 2967 X DBW 88 was found as desirable cross combination in $\mathrm{E}_{3}$ environment as it showed desirable (significant negative) inbreeding depression for grain yield per plant and some other traits.

\section{Introduction}

Wheat one of the most important food crop and cereal crop remains the third most produced food grain in world a cereal crop. There is noteworthy production in the production and productivity of wheat but still it needs much more improvement the yield and quality of wheat as it is a staple good of more than one billion people and still the population is burgeoning. The fundamental objective of most of plant breeding programmes remain to increase yield of concerned crop by developing improved varieties through heterotic expression which is expected to decline in $\mathrm{F}_{2}$ generation due to decrease in heterozygosity and referred as inbreeding depression.

The estimates of heterosis and inbreeding depression together provide information about the type of gene action involved in the expression of various quantitative traits. The mating between more closely related individuals is known as inbreeding. The closest form of inbreeding is self-fertilization. 
The genetic effects of inbreeding on crossfertilizing crops are as follows (Falconer, 1981).

Homozygosity is increased which results in fixation of genes. The recessive deleterious genes come to the surface due to release of hidden genetic variability from heterozygotes. These recessives are exposed to natural selection.

The heterozygosity is reduced rapidly because inbreeding results in elimination of hybrids from a population and replaces them with pure types. Thus, inbreeding leads to purity of types.

The populations mean is reduced due to decrease in the number of hybrid genotypes, which have more number of dominant genes.

Genetic correlation increases between close relatives due to increase in the prepotency, which increases with homozygosity.

The variability, which is hidden in heterozygote, is made free through inbreeding. Thus, the total variability is increased without selection. With selection the variability is reduced towards the direction of selection.

The inbreeding depression refers to decrease in fitness and vigour due to inbreeding. The degree of inbreeding is measured by the inbreeding coefficient. Inbreeding depression results are due to fixation of unfavorable recessive genes in $\mathrm{F}_{2}$, while in case of heterosis the unfavorable recessive genes of one line or parent are covered by favorable dominant genes of other parent. The fixation of all favorable dominant genes in one homozygous line is impossible due to linkage between some unfavorable recessive and favorable dominant genes. In the present study
Inbreeding depression is studied and analysis under three environments viz. normal. Late and very late sown conditions which are indicated as $E_{1}, E_{2}$ and $E_{3}$ respectively.

\section{Materials and Methods}

On the basis of genetic diversity and their stability for different yield traits, ten diverse wheat varieties selected as the experimental material and crossed in diallel mating fashion excluding reciprocals to obtain $45 \mathrm{~F}_{1} \mathrm{~s}$ in rabi 2014-2015. In Rabi 2015-16 ten genotypes along with their $45 \mathrm{~F}_{1}$ 's and $45 \mathrm{~F}_{2}$ 's (obtained at IARI, regional station, Wellington, TN during kharif 2015) progenies were evaluated using Randomized Block in 3 replications over three environments created by three different dates of sowing viz. 15 Nov., 5 Dec. and 25 Dec. at Agricultural Research Farm of RARI, Durgapura.

Observations were recorded on days to heading (Days), days to maturity (Days), grain filling period (Days), plant height (cm.), flag leaf area $\left(\mathrm{cm}^{2}\right)$, number of tillers per plant, spike length $(\mathrm{cm})$, number of grains per spike, 1000-seed weight $(\mathrm{g})$, harvest index $(\%)$ and grain yield/plant (g). Mean values over selected plants were used for statistical analysis. The mean values of different $F_{1} s$ and $\mathrm{F}_{2} \mathrm{~S}$ for all characters were subjected for analysis of inbreeding depression as per cent increase or decrease in the mean value of $F_{2}$ over $\mathrm{F}_{1}$.

\section{Results and Discussion}

The heterotic expression normally decreases in $F_{2}$ generation as the dominance or dominance interaction effects dissipate in this generation due to reduced heterozygosity, resulting into inbreeding depression. In present experiment, significant inbreeding depression was reported for different traits in all the three environments. 
Int.J.Curr.Microbiol.App.Sci (2018) 7(7): 1986-1993

Table.1.1 Estimates of inbreeding depression

\begin{tabular}{|c|c|c|c|c|c|c|c|c|c|}
\hline & $\begin{array}{l}\text { Days to } \\
\text { Heading } \\
\text { E1 }\end{array}$ & $\begin{array}{l}\text { Days to } \\
\text { Heading } \\
\text { E2 }\end{array}$ & $\begin{array}{l}\text { Days to } \\
\text { Heading } \\
\text { E3 }\end{array}$ & $\begin{array}{c}\text { Days to } \\
\text { Maturity } \\
\text { E1 }\end{array}$ & $\begin{array}{l}\text { Days to } \\
\text { Maturity } \\
\text { E2 }\end{array}$ & $\begin{array}{c}\text { Days to } \\
\text { Maturity } \\
\text { E3 }\end{array}$ & $\begin{array}{c}\text { Grain } \\
\text { Filling } \\
\text { Period } \\
\text { E1 }\end{array}$ & $\begin{array}{c}\text { Grain } \\
\text { Filling } \\
\text { Period E2 }\end{array}$ & $\begin{array}{c}\text { Grain Filling } \\
\text { Period E3 }\end{array}$ \\
\hline P1XP2 & $-2.331 *$ & $2.990 *$ & 1.987 & -0.763 & -1.246 & -0.719 & 0.927 & -6.161 & $-26.583 * *$ \\
\hline P1XP3 & -0.962 & $3.856^{*}$ & 0.852 & -1.305 & -0.901 & -1.071 & 2.243 & -6.738 & $23.428 * *$ \\
\hline P1XP4 & -1.095 & $2.724^{*}$ & 2.243 & 0.055 & -1.223 & -1.460 & -1.442 & $-7.959 * *$ & $-60.290 * * *$ \\
\hline P1XP5 & 1.350 & $3.505^{*}$ & 1.658 & -0.524 & -1.488 & -1.060 & 2.969 & $-9.650 * *$ & $-19.378 * *$ \\
\hline P1XP6 & $3.084 * *$ & $2.288^{* *}$ & 1.429 & -1.240 & -1.282 & -1.852 & 0.120 & -7.388 & $-11.658^{*}$ \\
\hline P1XP7 & 1.396 & 2.451 & 1.105 & -1.378 & -1.250 & -1.873 & 2.005 & $-5.621^{*}$ & $-38.045^{* * *}$ \\
\hline P1XP8 & 4.000 & $3.197 *$ & -0.067 & 1.416 & -1.266 & -1.449 & 0.701 & -7.609 & -8.366 \\
\hline P1XP9 & -0.457 & 2.851 & 2.281 & 0.545 & 1.458 & -1.418 & 2.702 & $-7.822 * *$ & 1.042 \\
\hline P1XP10 & 0.826 & $1.871^{*}$ & 2.138 & 0.254 & -0.307 & -0.719 & -2.179 & -9.484 & $-25.908 * * *$ \\
\hline P2XP3 & $2.548^{*}$ & 2.570 & 1.657 & -0.992 & -0.904 & -0.364 & -0.306 & $-5.583^{*}$ & $-50.032 * * *$ \\
\hline P2XP4 & -1.608 & $3.962 *$ & 0.751 & -0.775 & 1.994 & -0.362 & 1.726 & -6.167 & $-47.209 * * *$ \\
\hline P2XP5 & -0.541 & $2.603 * * *$ & 2.139 & -0.182 & 2.609 & -2.290 & -2.547 & -5.379 & $-39.516 * * *$ \\
\hline P2XP6 & 0.172 & $2.767^{*}$ & 1.596 & 1.326 & 0.619 & -1.838 & 0.899 & $-6.982 *$ & $-45.004 * *$ \\
\hline P2XP7 & $2.416^{*}$ & 3.548 & 1.563 & 1.818 & 0.197 & -1.128 & 3.220 & -6.660 & -0.307 \\
\hline P2XP8 & -0.463 & 2.642 & $1.992 *$ & -0.353 & 2.849 & -1.103 & 3.500 & -4.949 & $-33.201 * * *$ \\
\hline P2XP9 & 0.573 & 2.857 & $3.191^{*}$ & -0.771 & -1.112 & -1.083 & -0.879 & -2.802 & -12.006 \\
\hline P2XP10 & 0.000 & $3.996^{* *}$ & 1.524 & -0.088 & $6.497 * *$ & -1.465 & 3.842 & -5.445 & 1.809 \\
\hline P3XP4 & -0.728 & 2.510 & 0.541 & -0.512 & 0.306 & -1.792 & 3.408 & -7.889 & $18.692 * *$ \\
\hline P3XP5 & 0.701 & 3.225 & 2.131 & 0.508 & 3.768 & -1.413 & 4.321 & -5.860 & 3.596 \\
\hline P3XP6 & 1.900 & 2.347 & 2.094 & 0.850 & 2.128 & -1.449 & -0.736 & $-5.323^{*}$ & -5.892 \\
\hline P3XP7 & 1.429 & 3.302 & 1.613 & -1.238 & 0.000 & & 2.342 & $-10.406^{* *}$ & -2.679 \\
\hline P3XP8 & -0.236 & $3.318^{*}$ & $2.122 *$ & -1.028 & -0.909 & -2.281 & 0.178 & & 1.364 \\
\hline P3XP9 & 0.420 & $3.391 *$ & $3.134 * *$ & -0.126 & 1.739 & -2.273 & $4.648^{*}$ & $-7.692 *$ & -2.714 \\
\hline P3XP10 & 0.126 & 2.780 & $2.162 *$ & $-4.810 * *$ & 0.000 & -2.239 & -0.494 & $-7.005^{*}$ & -11.901 \\
\hline P4XP5 & 1.809 & 2.724 & 0.992 & -1.235 & $3.395 * *$ & -0.692 & 2.158 & -5.670 & $15.862 * *$ \\
\hline P4XP6 & 0.299 & 2.161 & 1.093 & -0.838 & -0.901 & -1.034 & 3.593 & $-7.571 * *$ & $-80.092 * * *$ \\
\hline P4XP7 & -1.571 & 2.439 & 1.130 & 0.787 & -1.307 & 1.767 & 1.338 & -9.353 & $-67.012 * * *$ \\
\hline P4XP8 & 0.794 & 2.315 & 1.483 & -0.942 & 0.912 & -0.730 & -4.430 & -5.068 & -15.338 \\
\hline P4XP9 & 0.823 & 3.070 & 2.247 & -0.773 & -0.901 & -1.429 & 3.130 & -3.487 & $-37.281 * * *$ \\
\hline P4XP10 & 1.317 & 2.729 & 0.939 & -1.064 & 2.586 & & 3.017 & & $-62.695 * * *$ \\
\hline P5XP6 & -0.261 & 2.666 & 0.532 & 0.549 & 3.254 & $-1.370^{*}$ & 4.552 & -7.053 & $-71.734 * * *$ \\
\hline P5XP7 & -0.704 & 3.330 & 1.124 & 0.552 & -0.331 & -1.730 & -1.183 & $-7.662 * *$ & -8.501 \\
\hline P5XP8 & 0.885 & 2.315 & 1.656 & -0.811 & -0.926 & -0.690 & $5.969 *$ & $-6.927 * *$ & $-11.317^{* *}$ \\
\hline P5XP9 & 0.919 & 4.245 & 1.648 & -0.220 & -1.603 & -1.053 & 3.395 & $-9.255^{* *}$ & $-46.530 * * *$ \\
\hline P5XP10 & 1.377 & $5.508 * * *$ & 1.053 & 1.429 & $6.079^{*}$ & -0.678 & 2.050 & $-11.452 * *$ & $-40.261 * * *$ \\
\hline P6XP7 & 1.396 & $7.583^{* *}$ & 2.775 & 0.000 & -1.235 & -1.370 & 1.205 & -0.471 & $-45.740 * * *$ \\
\hline P6XP8 & -1.667 & $9.019 * *$ & 1.070 & -0.257 & -1.526 & -1.014 & 0.401 & -7.020 & 2.557 \\
\hline P6XP9 & -1.293 & $-7.263 * *$ & 1.413 & -1.282 & & -1.003 & $9.818^{*}$ & -5.065 & $-46.316^{* *}$ \\
\hline P6XP10 & -0.661 & 3.982 & 1.905 & 0.251 & 2.286 & -0.304 & $-8.004^{*}$ & $-7.535^{*}$ & $-43.158 * *$ \\
\hline P7XP8 & -1.708 & 1.990 & 2.968 & -1.676 & -0.965 & -0.699 & $17.813^{* *}$ & $-11.110 * *$ & -3.326 \\
\hline P7XP9 & -0.008 & 3.389 & 1.124 & 0.741 & 0.872 & -1.481 & 9.411 & -5.149 & $-13.444 * *$ \\
\hline P7XP10 & -1.716 & $5.136^{*}$ & 2.377 & -1.667 & -0.664 & -1.429 & 0.784 & -4.208 & $-23.001 * *$ \\
\hline P8XP9 & -0.400 & 2.279 & 1.657 & 0.249 & 0.301 & -1.056 & 4.488 & -6.765 & $-40.173^{* *}$ \\
\hline P8XP10 & -0.707 & 2.358 & 2.551 & -0.826 & -0.619 & -1.515 & 3.119 & -8.397 & $-26.707 * *$ \\
\hline P9XP10 & 0.172 & 2.290 & 1.124 & -1.312 & 2.882 & -1.465 & -2.458 & $-6.596^{*}$ & $-28.185^{* * *}$ \\
\hline Average & 0.251 & $3.032 * * *$ & $1.666 * * *$ & -0.400 & 0.569 & $-1.186^{*}$ & 2.078 & $-6.715 * * *$ & $-19.955 * * *$ \\
\hline
\end{tabular}


Table.2 Estimates of inbreeding depression

\begin{tabular}{|c|c|c|c|c|c|c|c|c|c|}
\hline & $\begin{array}{l}\text { Plant Height } \\
\text { cm E1 }\end{array}$ & $\begin{array}{c}\text { Plant } \\
\text { Height } \mathrm{cm} \\
\text { E2 }\end{array}$ & $\begin{array}{c}\text { Plant } \\
\text { Height } \mathrm{cm} \\
\text { E3 }\end{array}$ & $\begin{array}{c}\text { Flag Leaf } \\
\text { Area } \mathrm{cm}^{2} \mathrm{E} 1\end{array}$ & $\begin{array}{l}\text { Flag Leaf } \\
\text { Area } \mathrm{cm}^{2} \\
\text { E2 }\end{array}$ & $\begin{array}{c}\text { Flag Leaf } \\
\text { Area } \mathrm{cm}^{2} \\
\text { E3 }\end{array}$ & $\begin{array}{c}\text { Tillers/ } \\
\text { Plant E1 }\end{array}$ & $\begin{array}{c}\text { Tillers/ } \\
\text { Plant E2 }\end{array}$ & $\begin{array}{l}\text { Tillers/ } \\
\text { Plant E3 }\end{array}$ \\
\hline P1XP2 & 2.158 & -1.176 & $2.101^{*}$ & 7.540 & 4.352 & 8.083 & 14.286 & 6.129 & 14.170 \\
\hline P1XP3 & -1.534 & -1.008 & 0.608 & 5.849 & 1.744 & 7.485 & 18.875 & 16.780 & 7.909 \\
\hline P1XP4 & -0.676 & -1.772 & -1.366 & 13.834 & -2.959 & 10.551 & 50.000 & 13.913 & 29.923 \\
\hline P1XP5 & -1.093 & -2.518 & $3.496 *$ & 11.292 & 5.509 & 8.667 & 3.138 & 5.950 & 7.742 \\
\hline P1XP6 & -0.313 & -0.658 & $3.646^{*}$ & $13.766^{* * *}$ & 0.000 & 9.706 & 12.500 & 14.702 & -14.870 \\
\hline P1XP7 & -1.240 & -0.809 & -1.587 & 11.300 & 3.258 & -2.130 & 18.182 & 20.524 & 24.675 \\
\hline P1XP8 & 1.046 & -2.308 & 1.112 & $24.968 * * *$ & 4.555 & 10.521 & 8.333 & 25.208 & 18.153 \\
\hline P1XP9 & $3.368^{*}$ & -1.328 & -1.159 & $20.352 *$ & 1.752 & 10.194 & -28.571 & 15.067 & -1.732 \\
\hline P1XP10 & -1.417 & -1.219 & -2.114 & 14.356 & 3.865 & 1.390 & -24.388 & $14.610 *$ & 30.720 \\
\hline P2XP3 & 6.490 & -1.379 & 0.638 & 20.424 & 5.265 & -9.583 & -12.500 & 24.806 & 48.987* \\
\hline P2XP4 & $5.002 * *$ & $-15.641 * *$ & 1.558 & 17.286 & 1.940 & 1.931 & 10.048 & 16.747 & 12.309 \\
\hline P2XP5 & 3.948 & -3.115 & 0.959 & 13.945 & 16.357 & 10.563 & 24.471 & 21.585 & 24.620 \\
\hline P2XP6 & -1.426 & -2.253 & -0.210 & $9.904 * *$ & 3.470 & 8.073 & 8.056 & 27.654 & 0.645 \\
\hline P2XP7 & $-5.376^{* * *}$ & $-3.529 * *$ & -3.550 & 6.756 & -6.125 & -41.077 & -12.500 & 22.321 & 22.374 \\
\hline P2XP8 & $8.172 * * *$ & -1.849 & 1.200 & 17.881 & 4.522 & 5.306 & 7.619 & 13.255 & 24.348 \\
\hline P2XP9 & $3.266^{*}$ & -1.011 & -1.681 & $19.253 * *$ & 5.525 & -12.876 & 16.000 & 7.612 & 25.112 \\
\hline P2XP10 & $-11.358^{*}$ & -1.001 & -4.580 & 6.502 & 2.528 & 7.884 & 9.167 & 23.993 & 29.235 \\
\hline P3XP4 & -1.587 & -0.977 & -1.139 & 6.218 & 5.699 & 14.776 & 26.435 & 11.614 & 7.720 \\
\hline P3XP5 & $-12.207 * * *$ & -1.310 & -0.725 & 8.217 & 4.678 & -9.880 & -16.667 & 14.556 & -27.778 \\
\hline P3XP6 & $6.241 * * *$ & -1.447 & 0.949 & $27.332 * * *$ & 3.705 & -24.904 & $-37.619 *$ & 17.232 & 12.425 \\
\hline P3XP7 & -1.698 & -0.764 & -1.673 & 13.697 & 3.449 & 8.824 & -13.636 & 24.027 & 15.625 \\
\hline P3XP8 & 1.116 & -1.279 & 1.139 & 21.365 & 3.696 & 8.112 & 4.167 & 10.636 & 29.140 \\
\hline P3XP9 & -0.218 & -0.816 & 1.244 & $26.019 * *$ & 1.957 & 9.028 & 25.000 & 18.280 & 1.201 \\
\hline P3XP10 & 1.905 & -1.419 & 1.392 & 5.449 & 18.032 & 2.636 & -7.066 & 20.788 & 8.007 \\
\hline P4XP5 & -0.080 & -1.071 & 1.326 & -1.791 & 2.506 & 15.219 & 11.111 & 21.504 & -4.407 \\
\hline P4XP6 & 2.148 & -1.093 & -0.050 & $31.773 * * *$ & 12.504 & 9.107 & 8.333 & 9.097 & 28.314 \\
\hline P4XP7 & 0.133 & -1.081 & 1.375 & $22.235^{*}$ & 6.099 & 10.572 & -12.676 & 15.939 & 27.803 \\
\hline P4XP8 & -1.250 & -2.720 & 0.803 & 4.814 & 3.696 & 5.496 & -2.857 & 19.786 & 9.082 \\
\hline P4XP9 & 2.926 & -1.330 & 1.078 & -5.815 & 5.525 & 5.523 & 9.524 & 17.097 & -19.134 \\
\hline P4XP10 & 0.830 & -1.911 & 1.926 & -3.110 & 5.740 & -10.664 & 0.111 & 19.908 & 13.713 \\
\hline P5XP6 & 1.919 & -0.697 & 1.061 & $26.877 * * *$ & 3.292 & -18.535 & 0.000 & 15.476 & -10.819 \\
\hline P5XP7 & -0.806 & -0.606 & 1.983 & $33.148^{*}$ & 2.006 & 6.985 & 0.000 & 18.034 & 17.619 \\
\hline P5XP8 & 1.924 & -1.492 & 0.789 & $35.971 * *$ & 1.123 & -3.477 & 9.091 & 20.755 & 32.807 \\
\hline P5XP9 & $10.432 * *$ & -0.832 & -3.248 & $31.209^{* * *}$ & 11.376 & 2.282 & 0.000 & 15.647 & $26.667^{*}$ \\
\hline P5XP10 & $-10.161 * * *$ & -2.226 & -0.995 & 6.275 & 6.242 & -2.674 & 13.995 & 28.054 & 0.486 \\
\hline P6XP7 & -1.435 & -2.074 & -2.133 & 15.784 & 3.348 & -18.569 & -15.789 & 16.760 & 2.130 \\
\hline P6XP8 & $2.985^{* *}$ & -1.698 & -0.412 & 7.000 & 2.482 & -8.576 & 16.667 & 5.751 & 21.442 \\
\hline P6XP9 & 0.537 & -0.447 & -0.461 & 12.136 & 7.067 & -12.407 & 10.000 & 11.436 & -6.803 \\
\hline P6XP10 & $2.653^{*}$ & -0.287 & -9.301 & 1.729 & -4.602 & -14.912 & 14.729 & 18.636 & -2.644 \\
\hline P7XP8 & $2.188^{*}$ & -2.000 & 2.997 & $27.691 *$ & 2.390 & 10.256 & 7.407 & 17.032 & 29.243 \\
\hline P7XP9 & $3.975^{*}$ & -0.293 & -0.815 & 9.142 & 7.838 & 7.892 & 8.696 & 16.803 & 20.695 \\
\hline P7XP10 & $5.426^{* *}$ & -1.327 & 4.102 & $34.177 * *$ & 2.805 & 13.680 & -23.333 & 8.059 & 0.731 \\
\hline P8XP9 & $2.660^{*}$ & -0.972 & 0.077 & 8.447 & 8.046 & 10.302 & 21.466 & $29.466 * *$ & -2.002 \\
\hline P8XP10 & $4.593^{*}$ & -1.119 & -1.393 & $28.698 * * *$ & 3.462 & $10.465^{*}$ & 12.500 & 18.919 & $30.901 *$ \\
\hline P9XP10 & 1.117 & -1.492 & 1.052 & $-15.624^{*}$ & 6.210 & 10.156 & 16.123 & 22.910 & -2.136 \\
\hline Average & 0.909 & $-1.706^{*}$ & 0.099 & $16.627 * * *$ & $4.256^{*}$ & 2.649 & 4.573 & 17.115** $_{*}$ & ${ }_{* *}^{13.906 *}$ \\
\hline
\end{tabular}


Int.J.Curr.Microbiol.App.Sci (2018) 7(7): 1986-1993

Table.3 Estimates of inbreeding depression

\begin{tabular}{|c|c|c|c|c|c|c|c|c|c|}
\hline & $\begin{array}{c}\text { Spike } \\
\text { Length E1 }\end{array}$ & $\begin{array}{c}\text { Spike } \\
\text { Length E2 }\end{array}$ & $\begin{array}{c}\text { Spike } \\
\text { Length E3 }\end{array}$ & $\begin{array}{l}\text { Grains/ } \\
\text { Spike E1 }\end{array}$ & $\begin{array}{c}\text { Grains/ Spike } \\
\text { E2 }\end{array}$ & $\begin{array}{l}\text { Grains/ } \\
\text { Spike E3 }\end{array}$ & $\begin{array}{c}\text { 1000-grain } \\
\text { Weight (g) } \\
\text { E1 }\end{array}$ & $\begin{array}{c}\text { 1000-grain } \\
\text { Weight (g) } \\
\text { E2 }\end{array}$ & $\begin{array}{c}\text { 1000-grain } \\
\text { Weight (g) } \\
\text { E3 }\end{array}$ \\
\hline P1XP2 & -6.733 & 16.686 & $-21.949 *$ & 5.100 & 2.918 & -13.777 & 2.030 & 7.383 & $16.086 * *$ \\
\hline P1XP3 & $-21.846 * *$ & 21.891 & -19.501 & 1.480 & 1.607 & $-20.083^{*}$ & 1.736 & 4.909 & $22.558^{* *}$ \\
\hline P1XP4 & -19.412 & 12.519 & -8.767 & 1.722 & 2.568 & -9.479 & 4.535 & 6.484 & $19.922 * *$ \\
\hline P1XP5 & -5.640 & $19.243 * *$ & 18.400 & 2.941 & -1.994 & -9.255 & 1.402 & 5.979 & $18.431 *$ \\
\hline P1XP6 & -2.873 & 6.732 & 4.268 & $2.795^{*}$ & 1.714 & -12.405 & -0.094 & 7.189 & $16.424 *$ \\
\hline P1XP7 & -2.083 & 15.968 & 6.859 & 5.729 & 2.554 & $-18.894 *$ & 2.813 & 7.745 & 14.387 \\
\hline P1XP8 & $-13.932 *$ & 19.524* & -0.811 & $36.808 * * *$ & 1.598 & -7.713 & 2.268 & $7.007 *$ & $10.311^{*}$ \\
\hline P1XP9 & -28.390 & 13.921 & -9.971 & $-11.331 * *$ & 2.382 & $-11.270 * *$ & 5.391 & $8.384 *$ & $15.049 * *$ \\
\hline P1XP10 & $-37.309^{* *}$ & 4.488 & 4.744 & $-8.159 *$ & $-8.681 * *$ & -10.524 & 4.656 & 8.342 & $17.398 * *$ \\
\hline P2XP3 & -14.950 & $20.288^{*}$ & 15.671 & 0.041 & 1.476 & -9.803 & 5.688 & 8.372 & $18.780^{* *}$ \\
\hline P2XP4 & -8.811 & 9.748 & $21.420 * *$ & -4.826 & 3.042 & -8.296 & 2.440 & 9.998 & 18.298 \\
\hline P2XP5 & -27.702 & 12.056 & 3.477 & 3.872 ** & 2.385 & $-19.628^{* *}$ & $5.167 *$ & 8.719 & 10.901 \\
\hline P2XP6 & $-28.079 *$ & 2.837 & 0.187 & 3.667 & 2.134 & $-8.912 *$ & 3.547 & 7.872 & 17.295 \\
\hline P2XP7 & -7.447 & 7.309 & 11.830 & 11.440 & 2.090 & $-23.221 * *$ & 7.941 & 8.448 & $22.991 * * *$ \\
\hline P2XP8 & -31.011 & -4.813 & 13.060 & 0.208 & 3.241 & -8.347 & -8.011 & 5.946 & $15.406^{*}$ \\
\hline P2XP9 & -4.781 & 14.896 & 9.955 & -1.459 & 1.545 & -7.561 & 7.421 & 8.488 & $20.158^{*}$ \\
\hline P2XP10 & -12.349 & 10.509 & $28.350^{*}$ & -4.786 & -2.625 & $-18.946 * * *$ & 3.436 & 9.921 & $15.321 *$ \\
\hline P3XP4 & -4.647 & 2.856 & -3.374 & $19.658 * * *$ & 0.962 & -5.962 & 7.582 & 8.924 & 21.916* \\
\hline P3XP5 & 1.509 & 6.592 & -5.754 & 0.033 & 6.719 & $-10.034 *$ & 0.640 & $7.128^{*}$ & 15.431 \\
\hline P3XP6 & -14.867 & 15.042 & $-7.598 *$ & 2.121 & 1.480 & -5.519 & $4.429 *$ & $8.587^{*}$ & $16.321^{*}$ \\
\hline P3XP7 & -17.713 & $15.816^{*}$ & 14.368 & 3.472 & 0.650 & $-11.316^{*}$ & 1.991 & 10.360 & $21.568^{*}$ \\
\hline P3XP8 & -7.632 & 7.309 & 6.265 & 1.750 & 2.980 & $-9.880^{*}$ & 1.458 & 9.303 & $21.179^{*}$ \\
\hline P3XP9 & 1.464 & 13.067 & 13.723 & 2.150 & 2.708 & -11.450 & -2.076 & 6.444 & $16.011^{* *}$ \\
\hline P3XP10 & $-34.179 *$ & 0.102 & -14.587 & 1.935 & 1.408 & $-10.687 * *$ & 1.894 & 9.222 & $25.023 * *$ \\
\hline P4XP5 & -15.793 & $10.842 *$ & -6.996 & 3.842 & 2.130 & -6.130 & 5.811 & 5.607 & $12.225^{* *}$ \\
\hline P4XP6 & 10.617 & -2.443 & 10.797 & $3.605^{*}$ & 2.472 & -9.286 & $8.339 * * *$ & 7.201 & $18.190^{* *}$ \\
\hline P4XP7 & 2.865 & 7.731 & 7.145 & 3.332 & 2.189 & -9.351 & 1.048 & 6.039 & $15.769^{*}$ \\
\hline P4XP8 & 2.349 & 6.267 & 12.613 & 1.787 & 1.577 & $-9.138 * * *$ & 1.796 & 3.966 & 17.163 \\
\hline P4XP9 & $-21.450^{* *}$ & $23.830 * *$ & 8.798 & 1.998 & 3.519 & $-11.031 *$ & 3.225 & 8.945 & $18.061^{*}$ \\
\hline P4XP10 & $-47.558 * * *$ & 14.530 & $10.919 *$ & 2.088 & 3.841 & $-12.092 *$ & 2.167 & 8.617 & 17.832 \\
\hline P5XP6 & $-15.920^{*}$ & 14.244 & 11.271 & 2.277 & 1.729 & $-8.190^{*}$ & 3.986 & 2.580 & $25.689 * *$ \\
\hline P5XP7 & $-16.183^{*}$ & 3.363 & 10.113 & 1.861 & $-5.542 * *$ & -5.024 & 2.552 & 4.639 & $18.972 * *$ \\
\hline P5XP8 & $-25.321 *$ & $20.897^{*}$ & $23.830 * *$ & 1.840 & 2.516 & $-8.682 *$ & 4.647 & $8.762 *$ & 12.643 \\
\hline P5XP9 & -17.679 & 13.882 & 20.650 & 1.431 & 2.798 & $-8.488^{*}$ & 4.097 & 3.459 & $21.345^{* *}$ \\
\hline P5XP10 & -9.804 & 10.506 & 12.049 & $8.777^{* * *}$ & $-32.614 * * *$ & $-16.622^{*}$ & 1.024 & $9.598 *$ & $21.033^{*}$ \\
\hline P6XP7 & -11.547 & 6.454 & $33.212 * *$ & 1.750 & 3.038 & $-10.901 *$ & 7.230 & 4.866 & 19.159 \\
\hline P6XP8 & 11.398 & $18.070^{*}$ & $23.408^{*}$ & $3.960 *$ & 3.253 & -5.493 & 3.757 & 5.421 & 18.412 \\
\hline P6XP9 & -9.656 & $32.410^{*}$ & $21.252 * *$ & 3.391 & $19.469 * *$ & $-10.699 * *$ & 0.909 & $7.764 * * *$ & 16.346 \\
\hline P6XP10 & -7.887 & $21.300^{*}$ & $-31.840^{*}$ & 3.491 & 2.408 & $-17.038^{* *}$ & 3.169 & $6.379 *$ & $24.097 *$ \\
\hline P7XP8 & $-24.727 *$ & 2.990 & $76.100 * * *$ & 2.080 & -1.930 & $-10.720^{* *}$ & 2.645 & 6.527 & $22.545^{* *}$ \\
\hline P7XP9 & 13.282 & 14.262 & 28.045 & 3.708 & 3.637 & $-8.574 *$ & 6.506 & $8.227 *$ & $20.428 * *$ \\
\hline P7XP10 & 3.670 & -9.234 & $10.673^{*}$ & 2.207 & 0.620 & -7.186 & 2.180 & $8.331 *$ & $16.577^{*}$ \\
\hline P8XP9 & $-48.992 * *$ & $11.368^{*}$ & 15.776 & 1.763 & 3.584 & $-10.658^{* *}$ & 2.320 & 12.499 & $17.886^{*}$ \\
\hline P8XP10 & -6.554 & 16.147 & 6.220 & 1.385 & 1.503 & -8.402 & 3.505 & $6.545^{*}$ & $14.090 * *$ \\
\hline P9XP10 & $-21.177^{*}$ & $28.201 *$ & 21.858 & 2.137 & 2.524 & -5.540 & 2.319 & 8.266 & $17.556^{* *}$ \\
\hline Average & $-13.015 * * *$ & $12.037 * * *$ & $10.013 * * *$ & 3.178* & 1.332 & $-10.217 * * *$ & 3.181 & 7.391 & $17.957 * * *$ \\
\hline
\end{tabular}


Int.J.Curr.Microbiol.App.Sci (2018) 7(7): 1986-1993

Table.4 Estimates of inbreeding depression

\begin{tabular}{|c|c|c|c|c|c|c|}
\hline & $\begin{array}{r}\text { Harvest } \\
\text { Index } \\
(\%) \mathrm{E} 1\end{array}$ & $\begin{array}{r}\text { Harvest } \\
\text { Index }(\%) \\
\mathrm{E} 2\end{array}$ & Harvest Index (\%) E3 & $\begin{array}{r}\text { Grain } \\
\text { Yield/ } \\
\text { Plant } \\
\text { (g) E1 }\end{array}$ & $\begin{array}{r}\text { Grain } \\
\text { Yield/ } \\
\text { Plant (g) } \\
\text { E2 }\end{array}$ & $\begin{array}{r}\text { Grain } \\
\text { Yield } \\
\text { Plant }(\mathrm{g}) \\
\text { E3 }\end{array}$ \\
\hline P1XP2 & 1.340 & 3.168 & -7.945 & -0.222 & 10.13 & 8.634 \\
\hline P1XP3 & 2.230 & 3.120 & -6.937 & 5.994 & $22.07^{* *}$ & 3.102 \\
\hline P1XP4 & -4.229 & 2.055 & -10.056 & 7.097 & -7.4 & -14.309 \\
\hline P1XP5 & 5.271 & 1.549 & -6.888 & 1.944 & 13.85 & 10.711 \\
\hline P1XP6 & 3.994 & 3.067 & -7.713 & 8.154 & $13.83^{*}$ & 10.021 \\
\hline P1XP7 & 0.849 & 12.906 & -8.608 & 4.639 & 13.8 & 5.606 \\
\hline P1XP8 & 5.754 & 2.266 & -4.317 & 3.052 & $18.34^{* *}$ & 16.780 \\
\hline P1XP9 & 5.189 & 7.861 & $-12.067^{*}$ & -1.637 & $30.59^{* *}$ & 16.377 \\
\hline P1XP10 & 3.151 & 8.275 & $-8.715^{*}$ & -7.004 & $23.09^{* *}$ & $14.457 * *$ \\
\hline P2XP3 & -0.422 & 3.616 & -11.445 & $17.026^{*}$ & $22.67^{* *}$ & 22.737 \\
\hline P2XP4 & 4.308 & 4.489 & $-10.839 *$ & 9.540 & 14.55 & $17.089 *$ \\
\hline P2XP5 & -3.397 & 9.599 & -11.571 & 2.840 & $21.17^{*}$ & 14.313 \\
\hline P2XP6 & -3.814 & 7.343 & -7.808 & 12.150 & $22.88^{* *}$ & 20.707 \\
\hline P2XP7 & 1.947 & 9.040 & -11.466 & 10.020 & $20.44^{*}$ & $26.320 *$ \\
\hline P2XP8 & -1.658 & 4.793 & -9.037 & -4.045 & 13.96 & $18.239 *$ \\
\hline P2XP9 & 0.094 & $21.385 * *$ & -11.899 & 14.875 & $28.27^{* *}$ & 17.815 \\
\hline P2XP10 & 9.401 & -3.962 & -11.954 & -1.704 & 14.86 & 14.032 \\
\hline P3XP4 & 0.879 & $23.767 * *$ & -6.989 & 9.944 & $20.62^{* *}$ & 12.312 \\
\hline P3XP5 & 4.789 & $-20.958^{*}$ & $-8.226^{*}$ & 12.446 & 18.13 & $17.648^{* *}$ \\
\hline P3XP6 & 7.115 & $26.315^{* *}$ & -14.448 & 3.371 & 13.12 & 13.624 \\
\hline P3XP7 & -4.343 & -1.841 & $-12.233^{*}$ & -5.029 & $17.12^{*}$ & $22.134 *$ \\
\hline P3XP8 & 9.681 & 4.920 & -10.087 & -3.607 & $20.93^{* *}$ & 17.697 \\
\hline P3XP9 & 6.735 & 3.755 & -9.435 & 5.959 & $30.93^{* *}$ & 16.424 \\
\hline P3XP10 & -1.196 & -3.249 & $-10.202 *$ & -0.737 & $24.21^{* *}$ & 16.754 \\
\hline P4XP5 & -6.501 & -4.656 & -8.455 & 3.509 & 14.94 & 22.942 \\
\hline P4XP6 & 3.964 & $27.338 * * *$ & -0.069 & 2.859 & $14.35^{*}$ & 18.374 \\
\hline P4XP7 & 1.664 & 7.450 & $-12.890 *$ & 4.176 & 7.3 & 14.300 \\
\hline P4XP8 & -4.163 & $-15.907^{*}$ & $-11.788^{*}$ & 8.919 & 11.17 & 11.164 \\
\hline P4XP9 & 5.816 & -14.050 & -8.973 & 5.017 & $19.1^{*}$ & 10.532 \\
\hline P4XP10 & $11.772 *$ & 9.392 & $-10.309 *$ & 6.229 & 15.7 & 5.432 \\
\hline P5XP6 & 5.550 & 1.549 & $-10.704^{*}$ & 5.198 & $24.79^{* *}$ & 12.495 \\
\hline P5XP7 & 5.386 & $15.453 * *$ & $-11.860 *$ & -0.632 & 3.73 & 15.199 \\
\hline P5XP8 & $8.845^{* *}$ & $-11.261^{*}$ & $-17.996^{*}$ & 4.969 & $29.26^{* *}$ & $16.388 *$ \\
\hline P5XP9 & $8.042^{*}$ & $15.126^{* *}$ & -10.272 & 4.744 & $17.49^{* *}$ & $15.357 *$ \\
\hline P5XP10 & 3.623 & 0.273 & -9.086 & 0.441 & 11.79 & $28.010 *$ \\
\hline P6XP7 & 3.742 & 8.259 & -10.465 & 0.000 & $18.15^{*}$ & 18.599 \\
\hline P6XP8 & 1.500 & 1.544 & -3.695 & 10.920 & 16.46 & 22.946 \\
\hline P6XP9 & 5.092 & $17.733 * *$ & -8.223 & 6.623 & $18.34^{*}$ & 17.441 \\
\hline P6XP10 & 8.035 & -13.836 & $-6.613^{*}$ & 9.998 & 16.1 & $-25.012^{*}$ \\
\hline P7XP8 & 3.966 & $34.541 * *$ & -4.532 & 2.094 & 9.72 & $47.290^{* * *}$ \\
\hline P7XP9 & -0.740 & $-27.234^{*}$ & $-14.127 * *$ & 8.511 & $17.82^{*}$ & -8.964 \\
\hline P7XP10 & 7.639 & 7.444 & -16.321 & 8.633 & 5.97 & $18.600 *$ \\
\hline P8XP9 & -5.438 & $-11.047^{*}$ & -9.722 & -0.559 & 11.49 & 18.387 \\
\hline P8XP10 & 3.055 & $10.022 *$ & $-11.511 *$ & -2.837 & 9.97 & 18.199 \\
\hline P9XP10 & 3.540 & 2.601 & $-15.499 *$ & -3.023 & $20.99^{* *}$ & $19.118^{*}$ \\
\hline Average & 3.175 & $4.847 * * *$ & $-9.924 * * *$ & $4.243^{*}$ & $17.978 * * *$ & $14.967 * * *$ \\
\hline
\end{tabular}


Each cross exhibited significant inbreeding depression for at least one or more traits in one or more traits, however none of the cross exhibited consistency for each character over each environment. For example the cross Raj 3765 X HD 2967 for days to heading in $E_{1}$ and $E_{2}$ environments and plant height in $E_{3}$ environment showed desirable significant inbreeding depression but in other cases it showed inbreeding depression either in desirable direction but non-significant or in undesirable direction. Cross Raj $3765 \mathrm{X}$ DBW 621-50 for days to heading in $E_{2}$ environment, plant height in $\mathrm{E}_{3}$ environment and spike length in $E_{3}$ environment showed desirable significant inbreeding depression but in other cases it also showed inbreeding depression either in desirable direction but non-significant or in undesirable direction. The same pattern was displayed by other crosses also for exhibition of inbreeding depression. Such results were also reported for different crosses and characters. However the cross HD 2967 X DBW 88 displyed desirable (significant negative) inbreeding depression for grain yield per plant and some other traits viz. spike length $(\mathrm{cm})$ and number of grains per spike. Similar findings were also reported by Sharma and Menon (1996), Joshi et. al. (2003b), Prakash Ved and Joshi (2003), Singh et. al. (2004), Singh et al., (2012), Kumar and Kerkhi (2014), Kumar et al., (2015), Bhardwaj (2017) and Yadav 2017).

The negative inbreeding depression may result from the advantage of population buffering, which may occur in F2 generation due to the segregation of genes or sometimes because of formation of superior gene combinations, such a situation is valuable in conventional breeding programme.

\section{References}

Anonymous, 2015. Population growth (annual $\%)$. World Bank. Retrieved 20 January
2015.

Bhardwaj, R Genetic Studies for Yield and its Contributing Attributes in Bread Wheat (Triticum aestivum L. em Thell) Over Environments. Thesis (Unpub.) submitted to S. K. N. A. U. Jobner.

Falconer, D.S. 1981. Introduction to quantitative genetics, $2^{\text {nd }}$ ed. Longman, New York.

Grafius, J.E. 1959. Heterosis in barley. Agron. J., 51 (9): 551-554.

Grifing, B. 1956. Concept of general and specific combining ability in relation to diallel crossing system. Aust. J. Biol. Sci., 9 (4): 463-493.

Joshi, S.K., Sharma, S.N., Singhania, D.L. and Sain, R.S. 2003b. Hybrid vigour over environments in a ten parent diallel cross in common wheat. SABRAO. J. Br. Genet., 35: 81-91.

Kumar, A.; Harshwardhan; Kumar, A. and Prasad, B. (2015). Heterotic performance of diallel F1 crosses over parents for yield and its contributing traits in bread wheat. J. Hill Agri., 6 (1): 237-245.

Kumar, D. and Kerkhi, S.A. 2014. Heterosis studies for yield component traits and quality in spring wheat (Triticum aestivum L.). Supplement Genet. and Pl. Br., 9 (4): 1725-1731.

Prakash, V. and Joshi, P. 2003. Genetics of metric traits in spring wheat under normal and late sown environments. Crop Improv., 30 : 177-187.

Singh, H.; Sharma, S.N. and Sain, R.S. 2004. Heterosis studies for yield and its components in bread wheat over environments. Hereditas. 141: 106114.

Singh, V.; Krishna, R.; Singh, S. and Vikram, P. 2012. Combining ability and heterosis analysis for yield traits in bread wheat (T. aestivum L.).Indian J. Agric. Sci., 82: 11. 
Sharma, S.N. and Menon, U. 1996. Heterosis over environments in bread wheat. Crop Improv., 23: 225-228.

Yadav, J. 2017. Genetic architecture of yield and its associated traits in bread wheat (Triticum aestivum L. em. Thell.) over environments. Thesis (Unpub.) submitted to S. K. N. A. U. Jobner.

\section{How to cite this article:}

Ritu Choudhary, Hoshiyar Singh, Chhagan Lal and Darshita Bhat. 2018. Inbreeding Depression Analysis for Yield and Some of Its Associated Characters in Late Sown Condition in Bread Wheat (Triticum aestivum L.em. Thell). Int.J.Curr.Microbiol.App.Sci. 7(07): 1986-1993. doi: https://doi.org/10.20546/ijcmas.2018.707.234 